Case Report

\title{
Septic Embolic Stroke Followed by Hemorrhage and Brain Abscess in a Patient with Systemic Infections: A Case Report and Literature Review
}

\author{
Jama A. Mohamud, ${ }^{1}$ Jingtao $\mathrm{Wu}^{2}$ Ye Jing ${ }^{\mathrm{D}},{ }^{2}$ and Yu Wang ${ }^{3}$ \\ ${ }^{1}$ Department of Medical Imaging, Subei People's Hospital of Jiangsu Province, Medical College of Yangzhou University, \\ Yangzhou, China \\ ${ }^{2}$ Department of Medical Imaging, Subei People's Hospital of Jiangsu Province, Yangzhou University, Yangzhou, China \\ ${ }^{3}$ Department of Neurology, Subei People's Hospital of Jiangsu Province, Dalian Medical University, \\ Dalian, Liaoning Province, China \\ Correspondence should be addressed to Ye Jing; yzhyejing@163.com
}

Received 16 December 2017; Revised 25 February 2018; Accepted 26 March 2018; Published 3 May 2018

Academic Editor: Amit Agrawal

Copyright (C) 2018 Jama A. Mohamud et al. This is an open access article distributed under the Creative Commons Attribution License, which permits unrestricted use, distribution, and reproduction in any medium, provided the original work is properly cited.

\begin{abstract}
We report a case of 50-year-old man with a severe acute ischemic stroke followed by intracerebral hemorrhage and brain abscess due to systemic infection. His initial intracranial radiographic findings were normal but three days later MRI scan of the brain revealed well-defined rounded cystic lesion on the T2-weighted and T1-weighted images in the right basal ganglia; the lesion presented an area of diffusion restriction on DWI; lately the lesion was confirmed to be an early stage of cerebral abscess. A week later the patient was noted to have worsening neurological status and left extremity weakness, and emergency brain CT scan revealed massive intracerebral hemorrhage in the right occipital lobe; he underwent intracranial hematoma evacuation surgery. The hematoma was removed successfully, and the systemic infections were treated with antibiotics.
\end{abstract}

\section{Introduction}

Ischemic stroke is life-threatening and severe medical condition requiring urgent and comprehensive evaluation. Systemic infections can increase the risk of ischemic stroke and may worsen ischemic stroke (IS) prognosis. Acute infections of different types constitute a risk factor for IS, particularly within one week of the event $[1,2]$. We report here computed tomography $(\mathrm{CT})$ and the magnetic resonance imaging (MRI) findings and serial image changes in a case of an acute ischemic stroke followed by intracerebral hemorrhage and brain abscess caused by systemic infection; to the best of our knowledge, this has not been reported in the literature before.

\section{Case Report}

A 50-year-old man with a past history of hypertension and diabetes was referred to our hospital after the onset of leftsided numbness and weakness for 5 hours. The patient had not any other past medical history other than hypertension and diabetes, and there was no family history of similar diseases. A preoperative computed tomography (CT) of the head and CT angiogram of the brain (CTA) scan on admission showed no abnormalities and vascular malformations (Figures 1(a) and 1(b)). He was transferred to the intensive care unit (ICU) for further investigations and treatment. He was given an intensive care such as oxygen therapy and lipidlowering.

Initial examination at the time of admission showed a temperature of $39^{\circ} \mathrm{C}$, blood pressure of $140 / 92 \mathrm{mmHg}$, and a regular heart rate of 78/minute. His Glasgow coma scale (GCS) score was 7, pupils were equal and reacting, and there was grade 3 power in the left limb and grade 5 in the right limb. Further laboratory examination showed a leukocyte count of $16.5 \times 109 / \mathrm{L}$, a hemoglobin level of $129 \mathrm{~g} / \mathrm{L}$, neutrophil count of $83.4 \%$, blood gas of $\mathrm{pH}$ 7.463, PO2 88.4, PCO2 26.8, and glucose in urine 4+; his 


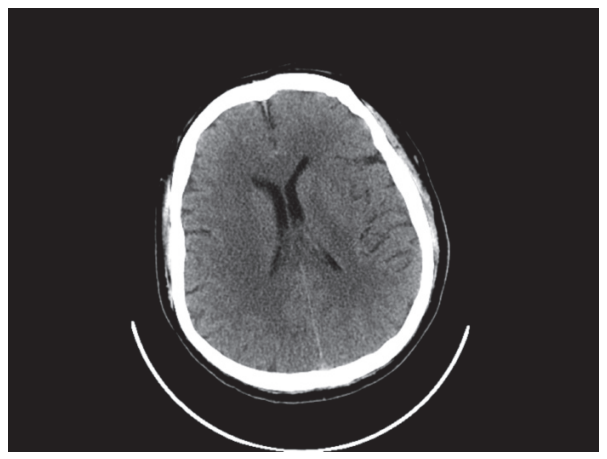

(a)

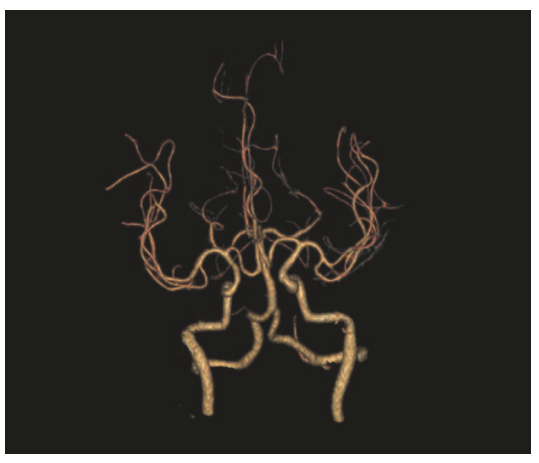

(b)

FIGURE 1: Initially, (a) a noncontrast computed tomography (CT) and (b) a computed tomography angiography (CTA) of the head performed on at the time of the admission showed no abnormalities and vascular malformations.

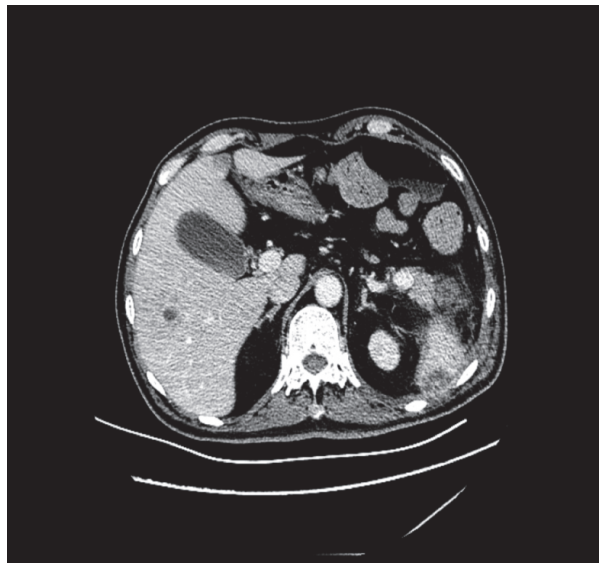

(a)

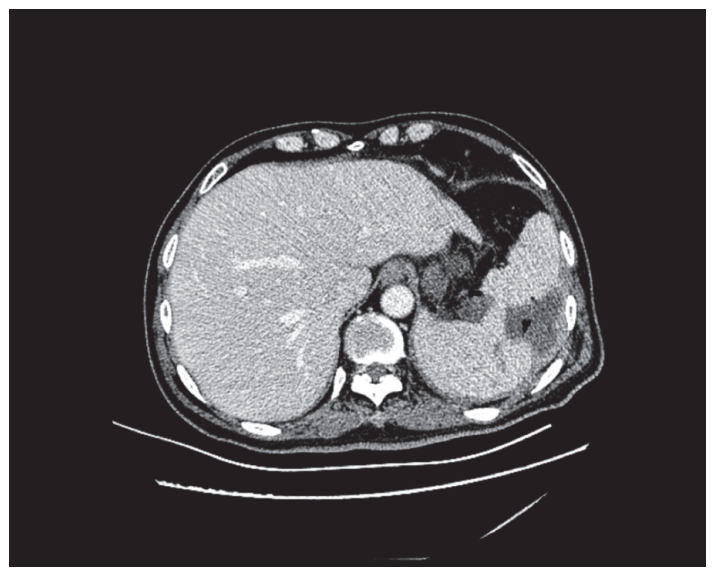

(b)

FIGURE 2: A contrast-enhanced CT scan of abdomen showed (a) rounded hypodense lesion with central low attenuation in the right lobe of the liver (suggestive of liver abscess) and (b) hypodense splenic air-filled lesion with an irregular wall (suggestive of perisplenic infection).

hypersensitive c-reactive protein was $497.4 \mathrm{mg} / \mathrm{L}$ (normal $<3 \mathrm{mg} / \mathrm{L})$. Echocardiography was performed showing a reduced compliance of left ventricle; the E-F slope of anterior mitral valve leaflet was decreased; otherwise, the size, structure, and movement of the heart were normal.

The patient was diagnosed with liver abscess, perisplenic and intracranial infection, and septicemia; a contrastenhanced CT scan of the abdomen showed rounded hypodense lesion with central low attenuation in the right lobe of the liver and a hypodense splenic air-filled lesion with an irregular wall (Figure 2). Ultrasonically guided percutaneous drainage of the splenic cyst fluid was performed; approximately $65 \mathrm{ml}$ of light yellow, murky fluid was removed. The blood cultures and splenic cyst drainage were positive with Klebsiella pneumoniae which were the primary source of septicemia.

On the third day in the hospital, magnetic resonance imaging (MRI) of the head showed high signal intensity on DW image and well-defined rounded cystic lesion on the T2-weighted and T1-weighted images involving in the right corona radiata and basal ganglia (Figure 3), although the lesion looks like that of an acute stroke but lately was confirmed to be early stage of cerebral abscess. On the seventh day, the patient was noted to have worsening neurological status and left extremity weakness and a repeat computed tomography (CT) scan revealed a right occipital lobe hematoma with blood loss of $40 \mathrm{ml}$ (Figure 4). He was immediately admitted to the surgical ward to undergo an urgent intracranial hematoma evacuation surgery. During the first three postoperative days the patient was delirious; further MRI of the brain three days after operation showed multiple hemorrhagic lesions involving both cerebral hemispheres and cerebellum and a cystic lesion in the basal ganglia region (Figure 5).

On hospital day 34, the patient developed high-grade fevers accompanied by worsening neurological status. Contrast-enhanced CT of the head showed a well-defined lesion, with thin, ring-like enhancement pattern in the right basal ganglia with perilesional edema, which compressed right lateral ventricle with midline shift towards left and was given the possibility of brain abscess (Figure 6). The patient was treated conservatively with supportive care; he was given 


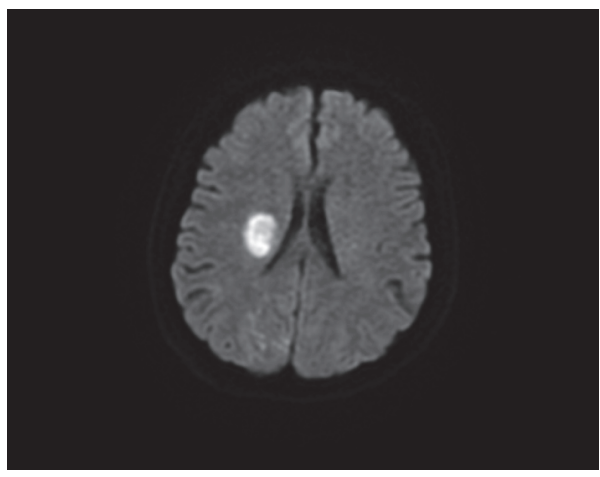

(a)

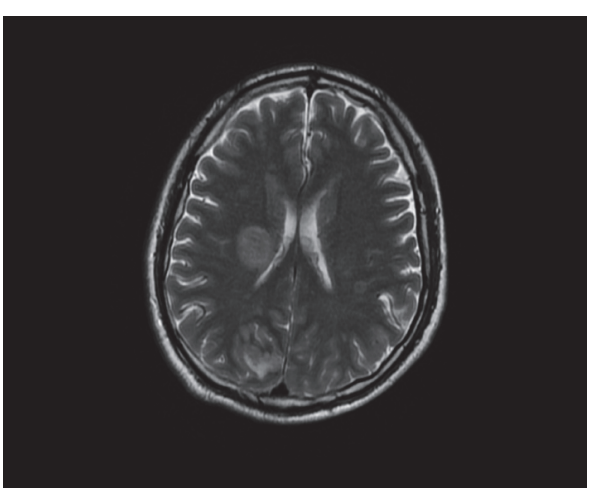

(b)

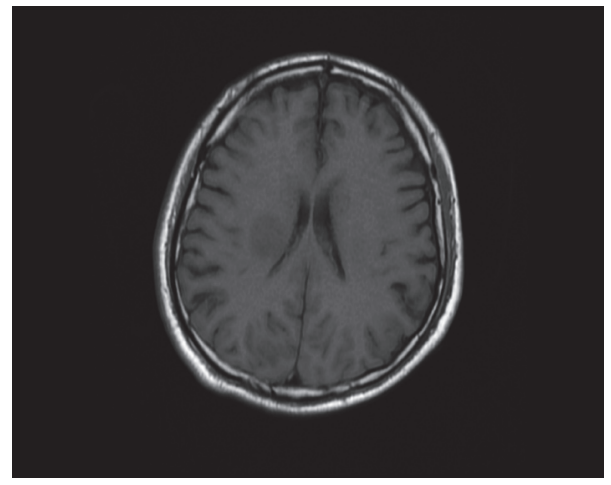

(c)

FIGURE 3: Brain MRI performed on the third day revealed (a) high signal intensity on DW image and (b, c) well-defined rounded cystic lesion on the T2-weighted and T1-weighted images involving in the right corona radiata and basal ganglia, compressing the right lateral ventricle with midline shift to left, suggestive of an early stage of brain abscess.

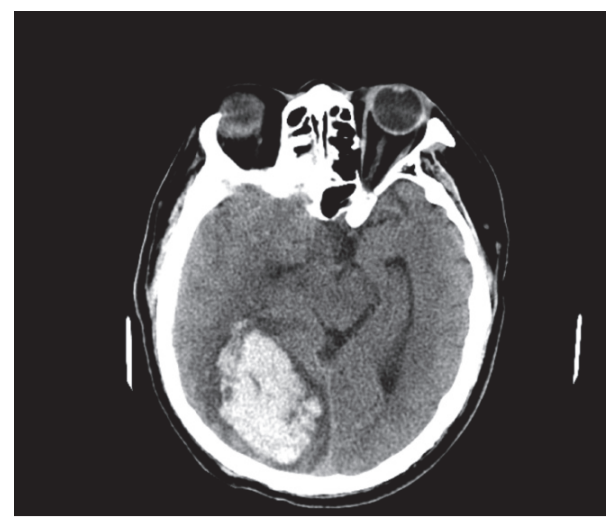

FIGURE 4: Noncontrast head CT performed on the 7th day demonstrates an acute hemorrhage in the right occipital lobe, with surrounding oedema and compressing the right lateral ventricle with midline shift to left.

an antiplatelet and anti-infective therapy; the antibiotic drugs that he was given were included imipenem, linezolid, and vancomycin. He was discharged to home after two months in stable condition and slow improvement in his mental status but he still had some weakness on the left side of his body.

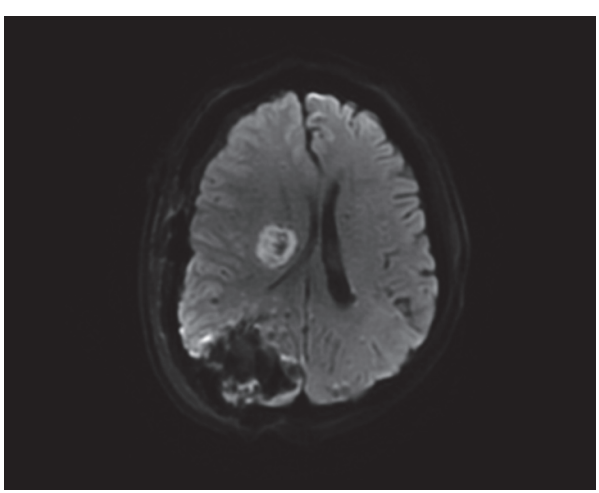

FIGURE 5: A contrast-enhanced MR imaging of brain performed three days after operation showed irregular mixed signal intensity on DWI with enhancement of its wall in the right occipital lobe and hyperintense lesion with small hypointense areas within in the basal ganglia region.

\section{Discussion}

Stroke has been defined as one of the most complicated pathologies; patients with stroke are at risk of developing a wide range of complications to their stroke, in particularly, stroke-associated infections increase the mortality and 


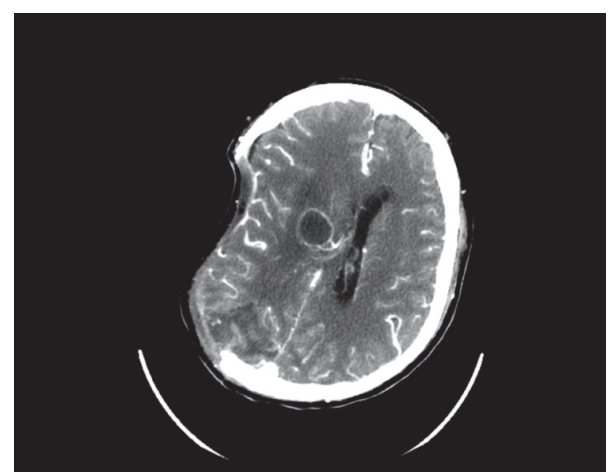

FIgure 6: Contrast-enhanced CT of head 30 days after the initial ischemia showed a ring-enhancing cystic lesion in the right basal ganglia with perilesional oedema, which compressed right lateral ventricle with midline shift towards left suggestive of abscess.

morbidity rates [1]. Acute ischemic and hemorrhagic stroke followed by brain abscess during the same hospitalization period is a rare entity and association of these three lifethreatening conditions may cause catastrophic result; it appears possible that acute infections of bacterial etiology, usually hepatic and of bacterial origin particularly in one week, may increase the risk factor for cerebral infarction in all age groups.

Septic embolic stroke usually results from vascular occlusion and corresponding degrees of ischemia and infarction, depending on vessel size, location, and collateral blood flow [3]. Cerebral arterial occlusion resulting in either infarction or transient ischemic attack accounts for $40-50 \%$ of central nervous system (CNS) complications of infective endocarditis [4]. The septic emboli are challenging because they involve three important cerebrovascular conditions: (a) cerebrovascular occlusions, (b) intracerebral abscess, and (c) arterial mycotic aneurysms. The main risk of neurologic complications is the absence of appropriate antibiotic therapy. It is important to note that most neurologic complications are already evident at the time of hospitalization or develop within a few days $[5,6]$.

The clinical course in the present case revealed unusual features and complex clinical manifestation. On his initial examinations, he was diagnosed with septicemia, liver abscesses, and perisplenic and intracranial infection indicating the presence of systemic infection and bacterial seeding in the body. Klebsiella pneumoniae was isolated from both the blood culture and splenic cyst drainage. Hence we hypothesize that hematogenous spread from the K. pneumoniae led to bacterial seeding of the ICH and the formation of the brain abscess, in the setting of impaired host defense mechanisms. The $k$. pneumonia bacteria entered the bloodstream and spread to the brain and thereby led to arterial thrombosis and the destruction of the vascular wall that resulted in right cerebral ischemia and the ICH; during the operation, we had seen multiple ruptured arterioles under the hematoma wall which was responsible for the bleeding vessels in the brain. This event illustrates that septic embolism can be considered as a cause of ischemic stroke and brain abscess in patients with a hepatic abscess.

Our patient was diabetic and hypertensive and did not have any other risk factors. Initial head CT scans done on admission time were normal. Three days later he developed acute ischemic stroke and brain abscess at different locations after an infectious complication. Cerebral abscesses are difficult to diagnose and they often mimic cerebral infarcts. In the earliest stages of cerebral abscess, a CT scan of the brain may be negative or it may show subtle nonspecific findings. However, MRI findings with a diffusion protocol are more helpful in differentiating cerebral stroke and abscess. Previous studies have reported that the formation of the cerebral abscess after a stroke is a rare event and serious condition that requires immediate recognition and treatment. However the need for having a high index of suspicion for septic stroke and cerebral abscess formation is necessary for early management, diagnosis, initiation of effective treatment, and prevention of significant morbidity and death $[7,8]$.

\section{Conclusion}

Intracranial hemorrhage after ischemic infarct due to septic emboli is a rare mechanism. K. pneumoniae is able to progress into severe bacterial infections leading to bloodstream infections and may lead to subsequent spread to brain parenchyma even in the absence of cyanotic heart disease. However, the recognition of infectious diseases and their risk factors for septic embolic stroke could be important for stroke prevention and may help to understand epidemiologic features of septic stroke.

\section{Conflicts of Interest}

The authors declare that they have no conflicts of interest.

\section{References}

[1] H. Emmez, A. Ö. Börcek, F. Doğulu, and N. Çeviker, "Ischemic stroke complicated by a brain abscess: A case report and review and review of the literature," Turkish Neurosurgery, vol. 17, no. 1, pp. 48-54, 2007.

[2] I. Y. Bova, N. M. Bornstein, and A. D. Korczyn, "Acute infection as a risk factor for ischemic stroke," Stroke, vol. 27, no. 12, pp. 2204-2206, 1996.

[3] S. P. Stawicki, M. S. Firstenberg, M. R. Lyaker et al., "Septic embolism in the intensive care unit," International Journal of Critical Illness \& Injury Science, vol. 3, no. 1, pp. 58-63, 2013.

[4] C. Özbek, U. Yetkin, M. Bademci, N. Karahan, and A. Gürbüz, "Ring annuloplasty and successful mitral valve repair in a staphylococcal endocarditis case with bilobular saccular mycotic aneurysm at cerebral artery and frontal region infarction. Secondary to septic emboli," Archives of Medical Science, vol. 4, no. 1, pp. 94-99, 2008.

[5] Magnetic Resonance Imaging of the Brain and Spine (2 Volume Set) Fourth Edition.

[6] R. Sonneville, B. Mourvillier, L. Bouadma, and M. Wolff, "Management of neurological complications of infective endocarditis in ICU patients," Annals of Intensive Care, vol. 1, article 10, 2011. 
[7] V. Bagga and M. Simons, "Cerebral abscesses—a stroke mimic," Age and Ageing, vol. 40, no. 5, pp. 645-645, 2011.

[8] L. A. Nadalo, Brain Abscess Imaging, J. G. Smirniotopoulos, Ed., MedScape, 2017. 


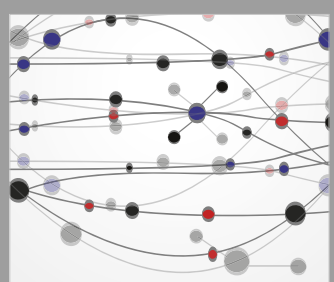

The Scientific World Journal
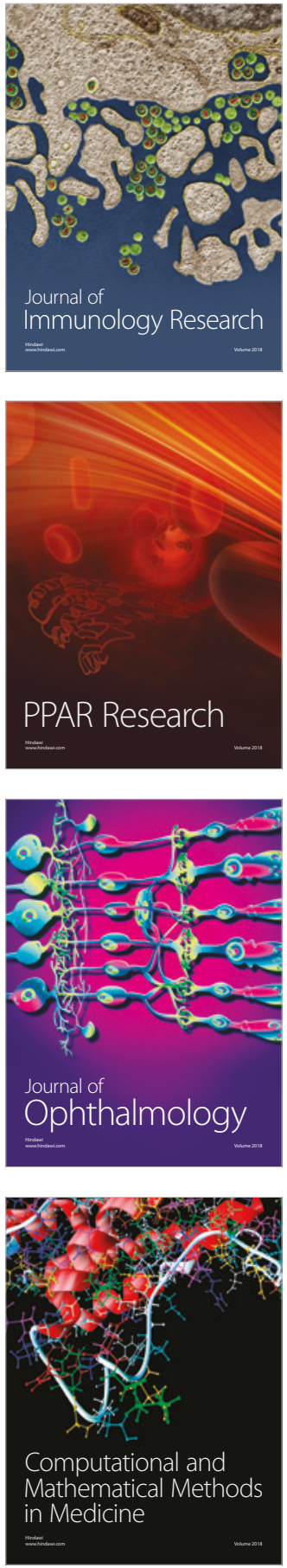

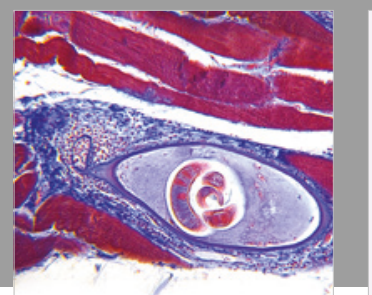

Gastroenterology Research and Practice

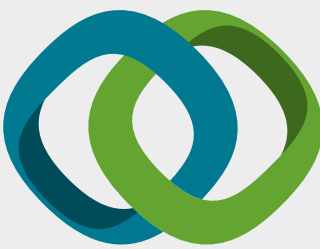

\section{Hindawi}

Submit your manuscripts at

www.hindawi.com
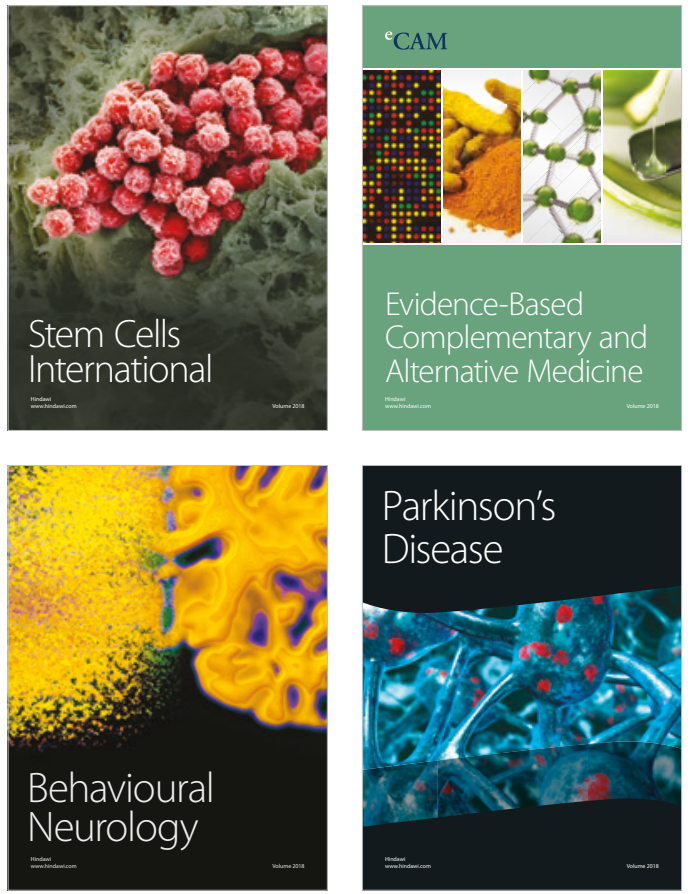

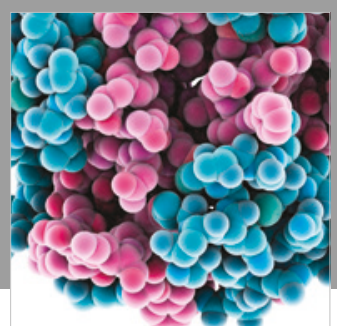

ournal of

Diabetes Research

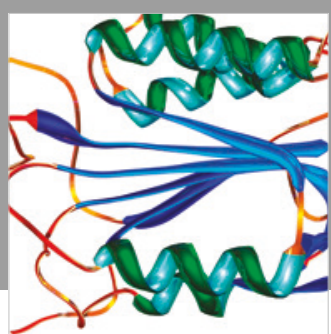

Disease Markers
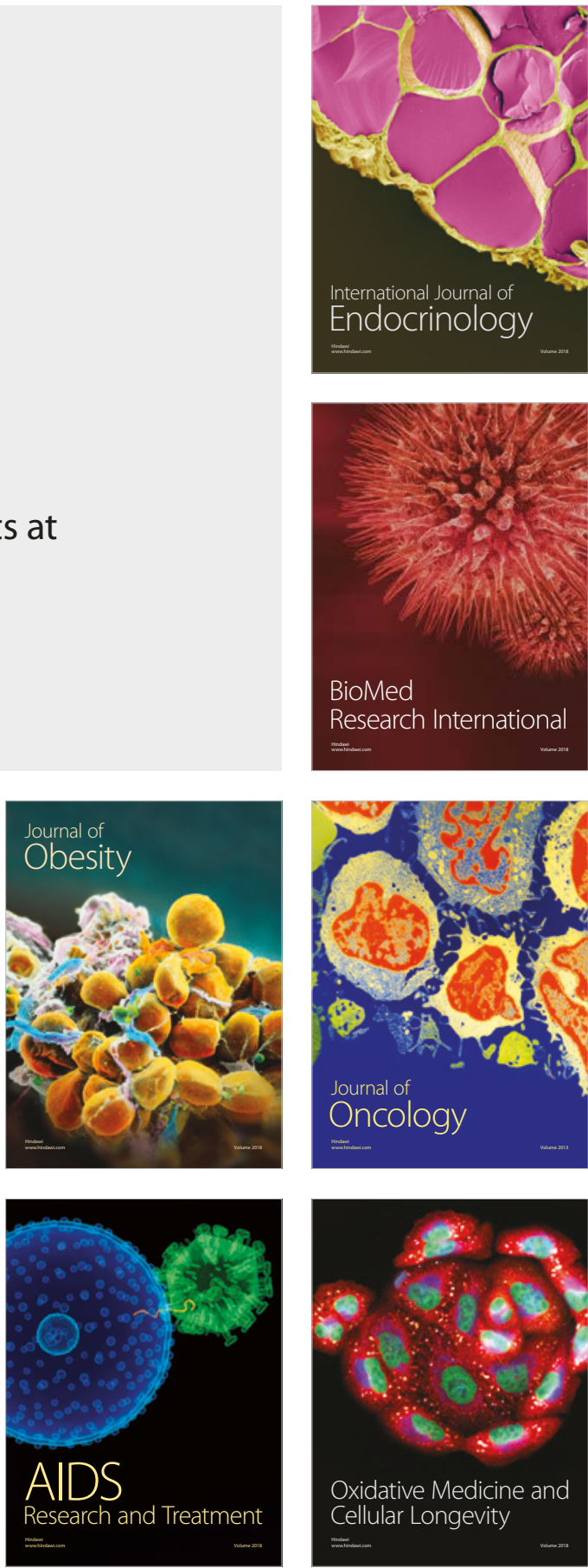